\title{
Modelling chemotherapy resistance in palliation and failed cure
}

\author{
Helen C. Monro ${ }^{\mathrm{a}}$, Eamonn A. Gaffney ${ }^{\mathrm{b}, *}$ \\ a School of Mathematics, University of Birmingham, Edgbaston, Birmingham B15 2TT, UK \\ b Centre for Mathematical Biology, Mathematical Institute, 24-29 St. Giles', University of Oxford, Oxford OX1 3LB, UK
}

\section{A R T I C L E I N F O}

\section{Article history:}

Received 22 July 2008

Received in revised form

12 November 2008

Accepted 2 December 2008

Available online 11 December 2008

Keywords:

Mathematical modelling

Drug resistance

Drug scheduling

Advanced cancer

Survival time

\begin{abstract}
A B S T R A C T
The goal of palliative cancer chemotherapy treatment is to prolong survival and improve quality of life when tumour eradication is not feasible. Chemotherapy protocol design is considered in this context using a simple, robust, model of advanced tumour growth with Gompertzian dynamics, taking into account the effects of drug resistance. It is predicted that reduced chemotherapy protocols can readily lead to improved survival times due to the effects of competition between resistant and sensitive tumour cells. Very early palliation is also predicted to quickly yield near total tumour resistance and thus decrease survival duration. Finally, our simulations indicate that failed curative attempts using dose densification, a common protocol escalation strategy, can reduce survival times.
\end{abstract}

(c) 2009 Elsevier Ltd. All rights reserved.

\section{Introduction}

The interest in modelling chemotherapy protocols to support clinical insight is driven by efforts to improve outcomes by adjusting drug scheduling (Browder et al., 2000; Citron et al., 2002; Fallik et al., 2003; Midgley and Kerr, 2005) and is illustrated by the seminal work of Norton and Simon $(1976,1986)$. This led to their initial hypothesis, namely that chemotherapy results in a rate of regression in tumour volume that is proportional to the rate of growth for an unperturbed tumour of the same size. Further work resulted in the influential concepts of dose intensification and especially dose densification. These constitute protocol escalation via either reducing rest phases, i.e. densification, or increasing drug dosage, i.e. intensification, and were predicted to increase the probability of tumour eradication (Norton, 1998, 1997, 2001). In particular the dose densification strategy was tested in a series of clinical trials by the Cancer and Leukaemia Group B (CALGB) and the American Breast Intergroup (Citron et al., 2002, 2003). The results supported the theory that dose dense treatment would lead to a significant improvement in clinical outcomes, additionally exemplifying how a mathematical model can make successful predictions for improved chemotherapy protocols (Piccart-Gebhart, 2003). This is further highlighted by recent phase I trials with a novel Capecitabine schedule for metastatic breast cancer (Tiffany et al., 2008). However, one

\footnotetext{
* Corresponding author.

E-mail addresses: monro@mat.bham.ac.uk (H.C. Monro), gaffney@maths.ox.ac.uk (E.A. Gaffney).
}

should also note that, in general, regimens utilising protocol escalation, either by dose densing or intensification, yield variable results (Piccart et al., 2000).

The focus of Norton and Simon's modelling strategies was to maximise cell kill; other objectives have been considered in the literature. For example, whereas Norton and Simon neglect genetic mutation to resistance, Goldie and Coldman (Goldie et al., 1982) modelled chemotherapy scheduling with the goal of minimising the development of drug resistance. Resistance was assumed to be a result of random genetic mutation to a resistant state (Goldie and Coldman, 1998). This assumption was based on the Nobel Prize winning work of Luria and Delbruck (1943), who showed that bacterial cultures developed resistance to bacteriophages at random. Spontaneous mutation has also been found to arise in in vitro cancer cell systems (Jaffrezou et al., 1994; Summer and Handshumacher, 1973), and Law (1952) later found that the same applied to methotrexate resistance in vivo with leukaemic L1210 cells.

The Goldie-Coldman model focuses on maximising the probability that no tumour cells will have mutated sufficiently to become resistant to all drugs present. When more than one non-cross-resistant chemotherapeutic is used, it was predicted that the drugs should be alternated as quickly as possible in order to reduce the occurrence of resistant cells, thus maximising the probability of cure (Goldie et al., 1982). Evidence supporting this hypothesis has been sought in numerous clinical trials (De Placido et al., 1995; Sieber et al., 2002; Siodlak et al., 1990), though it is typically refuted rather than validated. Gaffney $(2004,2005)$ extended the Goldie and Coldman model to consider cell cycle phase specific drugs and the effects of drug delivery. It was shown 
that the Goldie and Coldman's alternation hypothesis often breaks down both due to the effects of pharmacokinetics and due to resonances between the application time of a cell cycle phase specific drug and the tumour cell cycle time.

However, such models focus on cure, as do virtually all the models in the literature. In contrast, we will investigate the consequences of protocols when tumour eradication does not occur, either in the context of palliation or failed cure, and whether this reveals the need to consider different protocol strategies.

In particular, models have traditionally predicted that an increased dose will yield an increased response, at least within any implicit or explicit toxicity constraints. However, lower doses of chemotherapy may indeed lead to longer survival outcomes in the absence of cure. The failure of an aggressive chemotherapy schedule could occur due to the preferential removal of sensitive cells, leaving behind a population more resistant as a whole. At extremes, a non-curative but escalated chemotherapeutic regimen could be expected to leave behind an uncontrollable mass of cells insensitive to any further therapy. In contrast, too low a dose will allow even cells that are sensitive to the drug to grow out of control. Therefore we immediately have the question of whether one can reasonably anticipate that an intermediate level of chemotherapy will restrict tumour growth and increase survival duration in the palliative setting.

Protocol escalation is in conflict with the above reasoning, as escalation requires either a higher dosage of chemotherapy, a shorter administration time, or both. The source of this disagreement arises from the different objectives. The aim of protocol escalation is to improve the chances of eradicating the tumour; our above reasoning is based upon prolonging survival when tumour eradication is not possible. However, it cannot always be certain whether a tumour is curable or not. This emphasises that the effect of an attempted, but unsuccessful, curative protocol escalation on survival time also needs to considered in modelling chemotherapy scheduling and we will investigate this in detail.

We note that there is experimental evidence supporting the concept that lower chemotherapy doses could be preferable in the presence of resistance. In particular an investigation by Aabo et al. (1994) showed that low-dose chemotherapy delayed the relapse of a dominated and resistant sub-population in a human small-cell lung cancer (SCLC) xenograft in mice. The xenograft consisted of an artificially mixed BCNU-sensitive, dominating sub-population and a BCNU-resistant, undetectable (dominated) sub-population, where BCNU is an anti-cancer drug. At the time of tumour regrowth after low-dose treatment, most of the tumours continued to be dominated by the sensitive population, and thus remain susceptible to chemical control. At the time of regrowth after the response to high-dose treatment, the resistant cell line was the predominant population.

Similar concepts to those presented in this paper have been detailed in the modelling study of Hahnfeldt et al. (2003) with particular emphasis on the anti-angiogenic effects of metronomic chemotherapy, which is a low-dose drug scheduling regime. Such regimens are observed to differentially target the endothelial cells of the growing blood vessels found in tumours (Bello et al., 2001; Bertolini et al., 2003; Browder et al., 2000; Kerbel and Kamen, 2004; Man et al., 2002), therefore inhibiting angiogenesis. The model of Hahnfeldt et al. (2003) focuses on how heterogeneity in resensitisation rates between tumour and endothelial cells can provide a logical framework explaining such observations. Our model has an analogous mathematical framework though the central focus here is tumour cell heterogeneity per se rather than the difference between endothelial and tumour cells. This variation is primarily one of model interpretation; the core difference is that Hahnfeldt et al.'s model considers exponential cell growth. In contrast, we consider Gompertzian tumour growth; this is a constant, exponential, retardation of the growth rate which has been found to provide a good empirical description of the decelerating growth curves exhibited by more advanced tumours (Simpson-Herren and Lloyd, 1970; Sullivan and Salmon, 1972). The Gompertz (1825) model was first applied in actuarial statistics, and subsequently in the study of growth by Winsor (1932), with Laird (1964) further illustrating that the growth for a variety of primary and transplanted tumours in the mouse, rat and rabbit satisfied the Gompertzian relation.

Gompertzian growth is critical in this paper as it provides the theoretical basis of protocol escalation (Norton, 1997) in addition to being highly relevant for advanced tumours (Simpson-Herren and Lloyd, 1970; Sullivan and Salmon, 1972). For example, reducing a protocol's rest phase entails that a Gompertzian tumour is growing at faster and faster rates at each drug application since each application yields a smaller tumour. By the eponymous Norton-Simon hypothesis this compounds a greater and greater cell kill effect. This compounding entails that protocol outcomes are anticipated to be very sensitive to the dose densing effect. Such dynamics thus need to be explicitly considered when investigating protocols involving advanced tumours and when examining the consequences of dose densing, or more generally protocol escalation, and is central to our model.

In Section 2, the development of the model for palliative chemotherapy applied to advanced tumour growth will be outlined. Two main sections ofresults and discussion will follow. In Section 3.1 we consider continuous chemotherapy for our initial investigation into the effects of protocol escalation in the palliative setting. In Section 3.2, we proceed to consider protocol escalation for cycles of drug administration interspersed with rest phases. This study is subsequently extended to the case where the tumour is only just incurable to allow us to investigate the relationship between survival time and protocol escalation for a failed curative attempt. Finally, we discuss our results and observations in Section 4.

\section{The model}

The assumptions of the model are:

- Tumour growth is represented by a continuous, Gompertzian, model.

- The effect of the chemotherapeutic on the sensitive cells is to induce a regression rate proportional to the unperturbed growth rate, in accordance with the Norton-Simon hypothesis (Norton and Simon, 1976).

- The chemotherapeutic induces sensitive tumour cell kill in proportion to the intensity of the administration.

- Mutation to resistance is Darwinian (Law, 1952; Luria and Delbruck, 1943) at a rate proportional to the growth rate of the tumour (Goldie and Coldman, 1998).

- Toxicity constraints are not explicitly considered.

The final assumption requires further comment. For continuous protocols we seek to reduce the dosages of chemotherapy, which can be reasonably considered to reduce toxicity. When considering protocols with rest phases, dose densification is also a feasible option as we have the freedom to reduce the rest phases. This, however, may potentially lead to intolerable toxicity; while this has been observed (Piccart et al., 2000), there is empirical evidence it also need not be the case (Citron et al., 2003; Simon and Norton, 2006). Thus, should we draw a conclusion that protocol escalation via dose densification yields gains, it is, like previous work on this topic, ultimately qualified by an assumption 
that dose densing does not generate intolerable toxicity. However, should the modelling predict that dose densification is inappropriate even under the assumption that dose densing is tolerable, then the explicit inclusion of a toxic constraint will not alter this conclusion. Consequently, we can draw relevant and non-trivial conclusions without the complications of a toxic constraint.

The above assumptions lead to the following equations:

$\frac{\mathrm{d} N_{S}(t)}{\mathrm{d} t}=-\beta \ln \left(\frac{N(t)}{N_{\infty}}\right)\left[N_{S}(t)-\lambda C(t) N_{S}(t)+\tau_{2} N_{R}(t)-\tau_{1} N_{S}(t)\right]$,

$\frac{\mathrm{d} N_{R}(t)}{\mathrm{d} t}=-\beta \ln \left(\frac{N(t)}{N_{\infty}}\right)\left[N_{R}(t)+\tau_{1} N_{S}(t)-\tau_{2} N_{R}(t)\right]$,

where $N_{S}(t)$ and $N_{R}(t)$ represent the number of cells that are, respectively, sensitive and resistant to chemotherapy, and $N(t)$ represents the total number of cells. Let $N_{0}$ denote the initial number of tumour cells and let $N_{\infty}$ denote the theoretical value at which the Gompertzian tumour growth curve would saturate, although in reality this would be beyond the fatal level of the tumour. We also have the relation

$\beta \ln \left(N_{\infty} / N_{0}\right)=\alpha_{N_{0}}$,

where $\alpha_{N_{0}}$ is the instantaneous growth rate of the tumour at the start of the simulation and $\beta$ provides a measure of the rate of increase of the Gompertzian curve modelling tumour growth.

The parameter $\tau_{1}$ governs the rate of mutation of sensitive cells to resistance while $\tau_{2}$ governs the rate of back-mutation from resistance to sensitivity. There is a subtlety in the interpretation of our mutation rates, the discussion of which is deferred to the Appendix.

We now consider the chemotherapy term which consists of $\lambda$, a measure of the potency of the chemotherapeutic, and $C(t)$, the concentration of the chemotherapeutic agent at time $t$. Throughout the paper, $\lambda C(t)$ is either a non-zero constant, $C_{0}$, corresponding to the presence of chemotherapy, or zero, according to the details of the drug protocol and its scheduling. The effects of the chemotherapeutic are modelled by reducing $\beta$ for the sensitive cells via

$\beta N_{S}(t) \rightarrow \beta N_{S}(t)-\beta \lambda C(t) N_{S}(t)$,

with $N_{\infty}$ unaltered. This effective reduction in $\beta$ for the sensitive tumour cells, with $N_{\infty}$ fixed, is consistent with the growth curve measurements of Brünner et al. (1989) on comparing control and treated tumours. Additionally, the effect of the chemotherapeutic is proportional to the growth rate of the tumour and hence the model is explicitly in accord with the Norton-Simon hypothesis by design, and also with our assumption of linearity in the effect of the chemotherapeutic. Clearly the above representation of drug action does not incorporate cell cycle phase specificity, which requires tracking the cell cycle within the model. Finally, while one could in principle interpret Eq. (4) as entailing that the chemotherapeutic had an anti-proliferative effect we only consider the chemotherapeutic to upregulate cell death in the presentation below.

As detailed further in the Appendix, the simulations are initiated from a single sensitive seed, $N_{S}(0)=1, N_{R}(0)=0$. This seed is allowed to grow, producing a tumour with a mix of sensitive and resistant cells. Once this tumour is at a size consistent with the start of a palliative protocol, the chemotherapeutic protocol is switched on. The tumour cell number burden at chemotherapy initiation is defined to be $N_{c h}$. Unless stated otherwise, we take this to be

$N_{c h}=N_{\text {int }}^{0} \stackrel{\text { def }}{=} 10^{10}$ cells,

where $N_{i n t}^{0}$ corresponds to a tumour of radius of about $1.5 \mathrm{~cm}$ and is within the range of sizes reported for colorectal liver metastases at the start of palliative treatment (Becker et al., 1999). However, because the protocol start point is likely to be highly variable we do investigate the effects of altering the tumour cell burden at chemotherapy initiation.

The critical (fatal) level of the tumour is taken to be $N_{\text {crit }}$ cells, and the time at which this is reached is denoted $T_{\text {crit. }}$. The survival time $T_{s}$ is taken to be equal to $T_{\text {crit }}-T_{\text {int }}^{0}$, where $T_{\text {int }}^{0}$ is the time

Table 1

A list of representative, reference, parameters plus variables predicted by the model.

\begin{tabular}{|c|c|c|c|}
\hline Symbol & Interpretation & Value & Comment \\
\hline$N_{0}$ & Initial number of cells & 1 cell & $\begin{array}{l}\text { Tumour starts as one neoplastic cell, see } \\
\text { Appendix }\end{array}$ \\
\hline$N_{\infty}$ & Saturating number of cells in absence of therapy & $2 \times 10^{12}$ cells & Fatal level of uncontrolled growth, see Appendix \\
\hline$\alpha_{N_{0}}$ & Rate of initial exponential growth & $7 \times 10^{-3} \mathrm{~h}^{-1}$ & See Appendix \\
\hline$\beta$ & Rate of growth retardation & $2.47 \times 10^{-4} h^{-1}$ & See Appendix \\
\hline$\tau_{1}$ & Mutation to resistance per cell cycle & $1 \times 10^{-6}$ & Goldie and Coldman (1998) and Appendix \\
\hline$\tau_{2}$ & Back-mutation to sensitivity per cell cycle & $1 \times 10^{-6}$ & Goldie and Coldman (1998) and Appendix \\
\hline$N_{\text {crit }}$ & Critical (fatal) number of cells & $5 \times 10^{11}$ cells & Corresponds to a tumour diameter of $10 \mathrm{~cm}$ \\
\hline$N_{\text {int }}^{0}$ & Nominal, reference, number of tumour cells at treatment initiation & $10^{10}$ cells & $\begin{array}{l}\text { In range of palliation start points reported by } \\
\text { Becker et al. (Becker et al., 1999); see Appendix }\end{array}$ \\
\hline$N_{c h}$ & Actual number of tumour cells at therapy initiation & $10^{9}-3 \times 10^{11}$ cells & Varied, but equal to $N_{i n t}^{0}$ unless stated otherwise \\
\hline$\lambda$ & Chemotherapy potency parameter & & Varied \\
\hline$C(t)$ & Concentration of chemotherapy & & Varied \\
\hline$C_{0}$ & Level of chemotherapy cell kill & $0-40$ & Varied \\
\hline$N_{c y c}$ & Number of cycles in a drug protocol & & Varied. See Section 3.2 \\
\hline$t_{\text {rest }}$ & Rest phase duration in a drug protocol & & Varied. See Section 3.2 \\
\hline$T_{i n t}^{0}$ & Time for tumour to reach $N_{i n t}^{0}$ from initial seed & & Predicted \\
\hline$T_{\text {crit }}$ & Time taken to reach the critical (fatal) tumour burden & & Predicted \\
\hline$T_{s}$ & Survival time, $T_{\text {crit }}-T_{\text {int }}^{0}$ & & Predicted \\
\hline$T_{\max }$ & Maximum survival time on varying parameters such as $C_{0}, N_{c y c}$ or $t_{\text {rest }}$ & & Predicted; see Section 3. \\
\hline$T_{\text {lim }}, T_{0}$ & Survival time for most intense/dense protocol on varying parameters & & Predicted; see Section 3. \\
\hline$d_{c h}, A u C$ & Duration and area under curve of a continuous, time limited protocol & & Varied; see Section 3.1.2. \\
\hline$N_{S}, N_{R}$ & Sensitive, resistant tumour cell burden & & Predicted \\
\hline$N$ & Total tumour cell burden & & Predicted \\
\hline
\end{tabular}

Refer to the Appendix for further details of the parameter estimation. 
taken for the tumour burden to reach $N_{i n t}^{0}$ from the initial tumour seed in the absence of chemotherapy.

A summary of the parameters used in our simulations, together with reference values, are listed in Table 1 and further details on their estimation can be found in the Appendix. This table also lists variables predicted by the model, such as survival time.

By adjusting the drug protocol in the modelling appropriately, we can consider numerous palliative and failed cure chemotherapy regimens to investigate the effects of dose densification or other scheduling strategies on survival time when tumour control is lost. Given the model consists of a small number of ordinary differential equations, our use of an Euler method for numerical simulations is amply sufficient; there are no stability difficulties nor inconvenient computational overheads.

In Section 3.1 we focus on continuous palliative chemotherapy, for either a specified finite period or an indefinite infusion. The effects of the rate of cell kill, the size of the tumour at chemotherapy initiation and the duration of therapy on the survival time are investigated. In Section 3.2 regimens with a specified number of doses are simulated with varying rest phase durations. The resulting changes in the survival time are investigated, as are the effects of altering the rate of cell kill and the initiation time of chemotherapy.

\section{Results}

\subsection{Continuous chemotherapy}

\subsubsection{Indefinite continuous protocol}

Simulations of indefinite continuous chemotherapy are performed for different, fixed, values of cell kill $\lambda C(t) \equiv C_{0}$ with survival times depicted in Fig. 1. It clearly shows a local maximum, illustrating that an intermediate level of chemotherapy is predicted to prolong survival time the most.

Plots A, B, C of Fig. 2, respectively, show the cell population dynamics for the three values of cell kill, $C_{0}$, depicted by $A, B, C$ in Fig. 1. Note that in the optimal scenario, B, both sensitive and resistant cells are present in substantial numbers, illustrating the prediction that non-trivial tumour cell population competition is

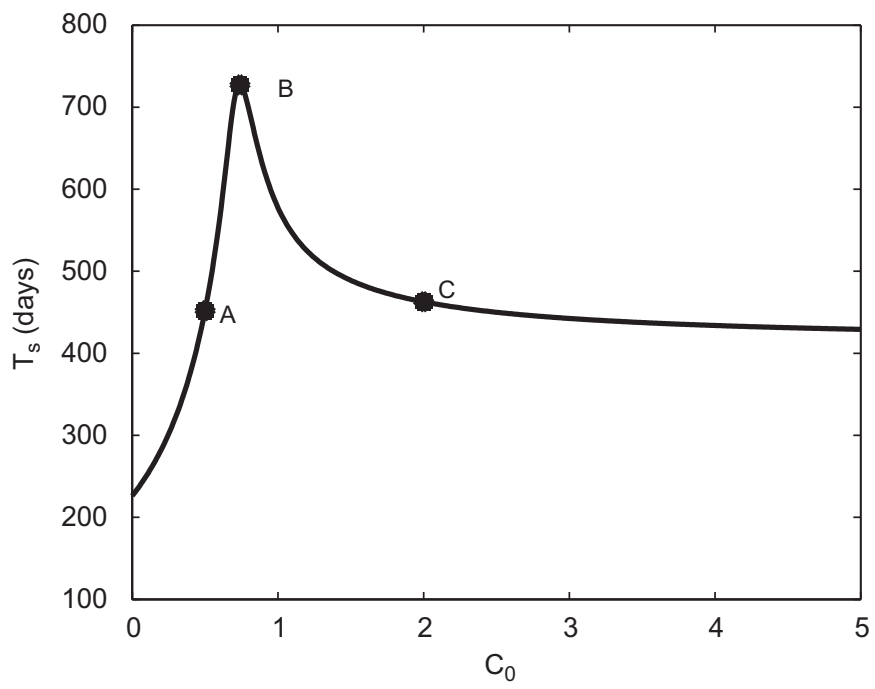

Fig. 1. Indefinite continuous chemotherapy, where $N_{c h}=N_{i n t}^{0}$. A plot of the survival time, $T_{s}$, for varying chemotherapy cell kill, $C_{0}$. The labels $\mathrm{A}, \mathrm{B}$ and $\mathrm{C}$ correspond to points chosen to represent the different behaviour of the cell populations for survival times below (A, corresponding to $C_{0}=0.74$ ), equal to ( $\mathrm{B}$, corresponding to $\left.C_{0}=0.9\right)$, and above ( $C$, corresponding to $C_{0}=2$ ) the maximum.
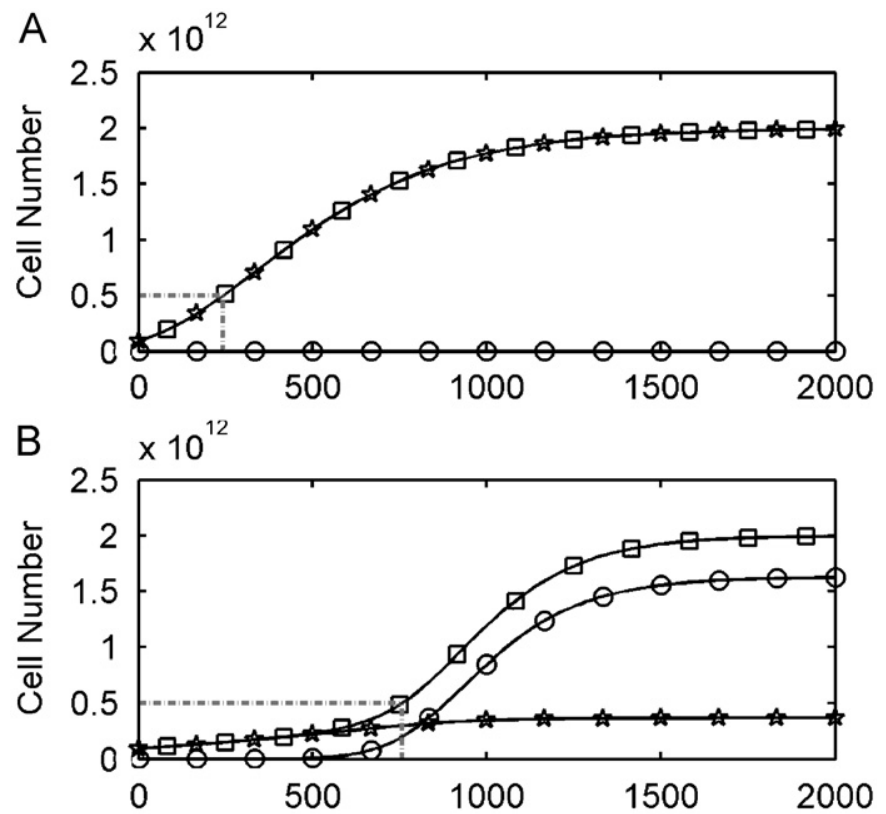

C $\times 10^{12}$

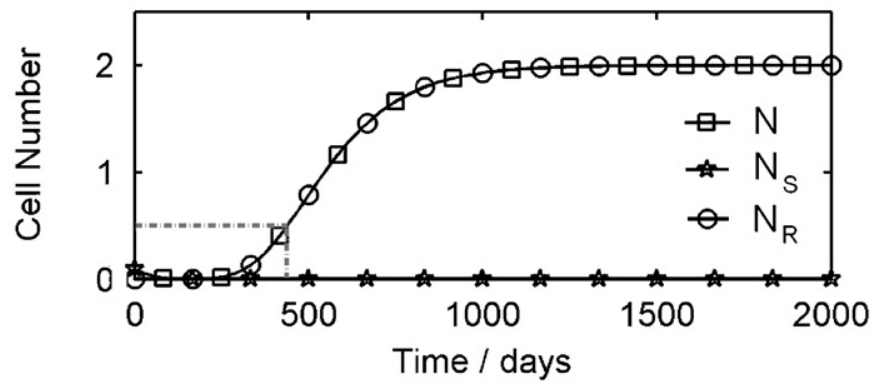

Fig. 2. Evolution of numbers of sensitive $\left(N_{S}\right)$, resistant $\left(N_{R}\right)$ and total $(N)$ cell populations during indefinite, continuous chemotherapy. The number of sensitive cells at chemotherapy initiation is approximately $1 \times 10^{10}$, and the number of resistant cells at this point is approximately $2 \times 10^{5}$. Plots $A, B$ and $C$ represent three different values of chemotherapy cell kill, $C_{0}$, corresponding to points $\mathrm{A}, \mathrm{B}$ and $C$ in Fig. 1. The dotted lines mark the point at which the tumour load reaches the fatal level of $N_{\text {crit }}$ cells.

present when the survival time is longest. Essentially, there is an optimal balance between the drug directly killing sensitive cells and indirectly promoting the production of resistant cells that determines the maximum survival time.

Due to variability in the likely start point of palliative treatment, we vary $N_{c h}$, the tumour cell burden at the start of chemotherapy. The survival time, $T_{s}$, once more the time to death from our reference tumour cell burden point of $N_{i n t}^{0}$ cells in the absence of chemotherapy, is plotted against cell kill, $C_{0}$, for different values of tumour cell number at the start of chemotherapy; see Fig. 3. Note the survival time is measured from this reference tumour cell burden point rather than the chemotherapy initiation time to give an absolute comparison between the regimes in terms of overall survival as opposed to survival following chemotherapy. The maximum survival times, denoted $T_{\max }$, from graphs such as those in Fig. 3 are plotted against the tumour cell number at chemotherapy initiation $N_{c h}$, in Fig. 4A. The value of the chemotherapy cell kill level which maximises the survival time is shown in Fig. 4D.

$T_{\text {lim }}$ is the limiting value of the survival time, $T_{s}$, as the sensitive cell kill tends to infinity. Hence $T_{\max }-T_{\text {lim }}$ measures how the survival time improves for the most judicious choice of dosing, 


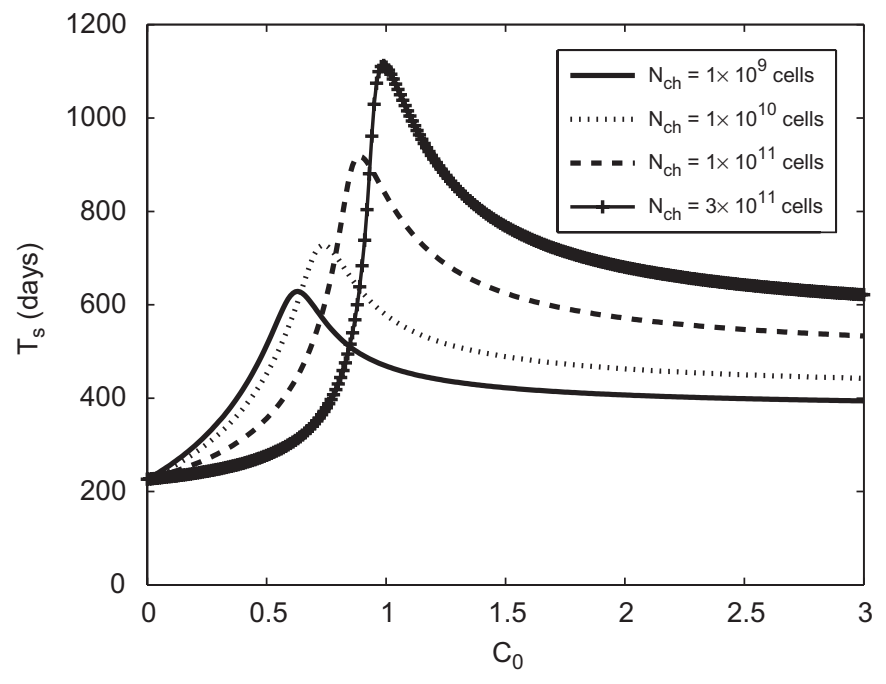

Fig. 3. Plots of the survival time, $T_{s}$, against cell kill, $C_{0}$, for indefinite continuous chemotherapy with different, fixed, tumour cell burdens at the start of chemotherapy, $N_{c h}$.
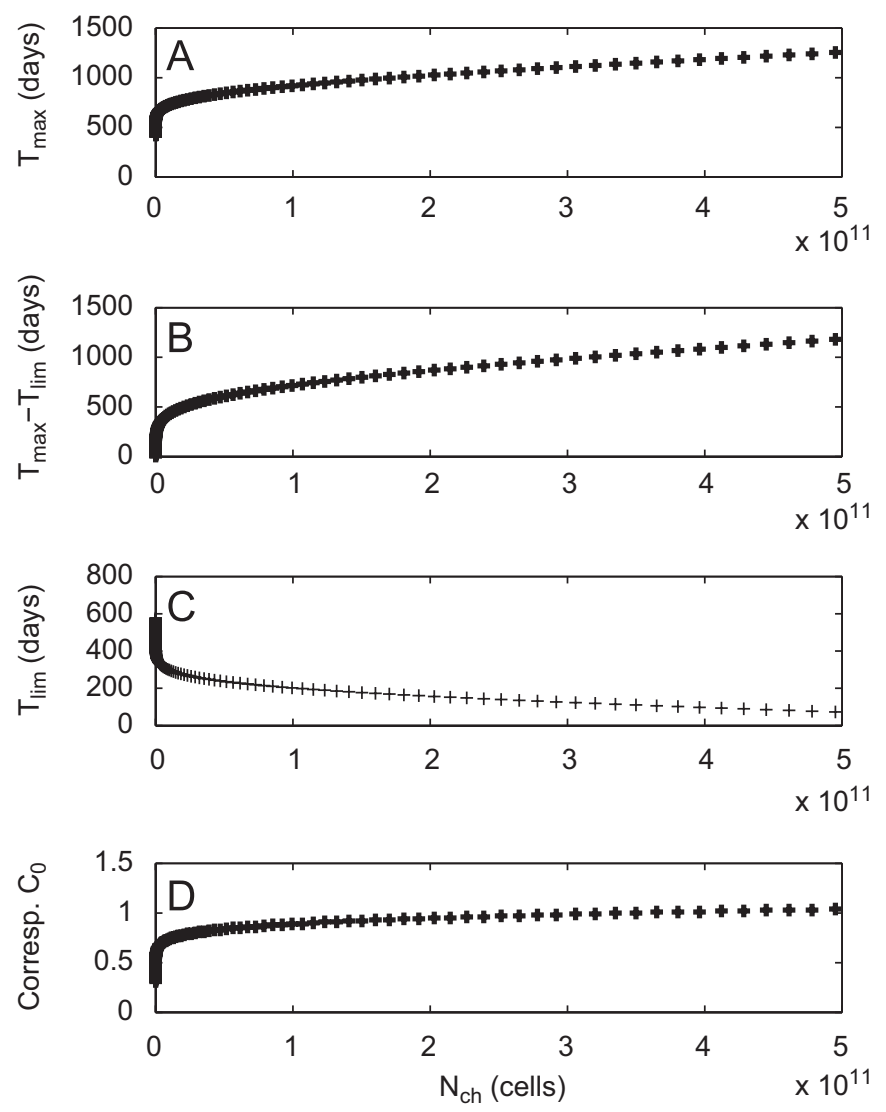

Fig. 4. Plot A shows the maximum survival time, $T_{\max }$, on varying the level of chemotherapy cell kill, $C_{0}$, for a range of the tumour cell number at the start of chemotherapy, $N_{c h}$. Plot B gives $T_{\max }-T_{\text {lim }}$, where $T_{\text {lim }}$ denotes limit of the survival time as the sensitive cell kill $C_{0}$ tends to infinity (shown in plot $C$ ). The difference $T_{\max }-T_{\text {lim }}$ therefore shows the difference in the survival time due to the most judicious choice of chemotherapeutic dosing compared to the theoretical maximum dose. Plot $\mathrm{D}$ shows the level of chemotherapy cell kill, $C_{0}$, corresponding to maximal survival time at each value of $N_{c h}$.

and thus cell kill, compared to the prediction in the limit of infinite sensitive cell kill. These are plotted with as the initial tumour burden varies in Fig. $4 \mathrm{~B}\left(T_{\max }-T_{\text {lim }}\right)$ and Fig. $4 \mathrm{C}\left(T_{\text {lim }}\right)$.
Note that the model predicts that survival time increases with delayed chemotherapy, at least providing the model is valid, i.e. that the increased chemotherapy dosing required for optimal control, and the increased tumour size do not exceed tolerable limits. However, the survival time then drops dramatically as the initial tumour cell number reaches $5 \times 10^{11}$ cells, simply because the initial tumour size approaches the fatal tumour size.

\subsubsection{Finite continuous chemotherapy}

Chemotherapy is usually administered for a fixed period of time, rather than ongoing indefinitely, so finite drug schedules are also considered. The start-time of the therapy is once more taken to coincide with a tumour cell burden of $N_{i n t}^{0}=10^{10}$ cells in the following sections. The intended chemotherapy duration, denoted $d_{c h}$, is then varied; the actual duration is cut short if the tumour cell number reaches the fatal limit during the protocol. Here the total amount of chemotherapy administered is considered since the schedules are finite; this is commonly represented as the "Area under (the) Curve", denoted $A u C$, of the treatment

$A u C=C_{0} d_{c h}$.

Survival time is plotted against $A u C$ for different values of therapy duration, $d_{c h}$, in Fig. 5. The maximum survival time for each $d_{c h}$, on variation of the area under curve, is plotted in Fig. 6A and denoted $T_{\max }$. Analogously to Fig. $4 \mathrm{C}, T_{\text {lim }}$ is the limit of the survival time as the chemotherapeutic sensitive cell kill, and thus the area under curve, tends to infinity; $T_{\max }-T_{\lim }$ is plotted in Fig. $6 \mathrm{~B}$. The values of $\mathrm{AuC}$ and $C_{0}$ corresponding to the maximal survival time are also shown.

Note the model predicts that for a fixed chemotherapeutic duration, an intermediate level of chemotherapeutic, as measured by the area under curve, produces optimal results and that this effect is emphasised for longer protocols. In addition, up to a threshold, we also have the prediction that increasing the duration of the protocol, with a concomitant reduction in the level of cell kill, can also increase survival time.

\subsection{Protocols with rest phases}

We proceed to consider protocol escalation when rest phases are present; in addition to dose intensification by increasing chemotherapeutic cell kill, we can also investigate dose densification by reducing the rest phase duration.

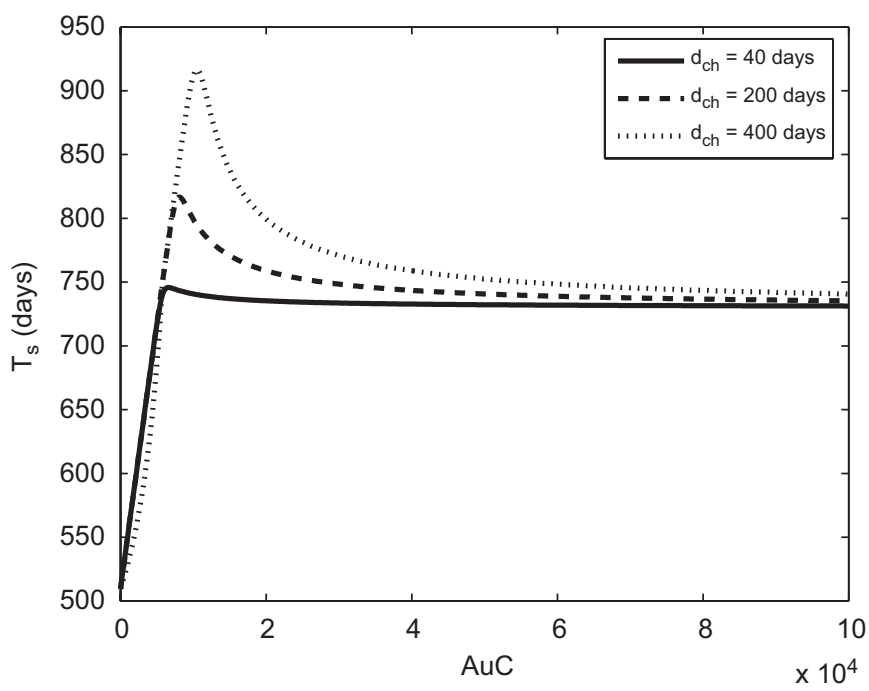

Fig. 5. Finite continuous chemotherapy. The survival time, $T_{s}$, is plotted against the area under curve, $A u C$, for different values of chemotherapy duration $d_{c h}$. 

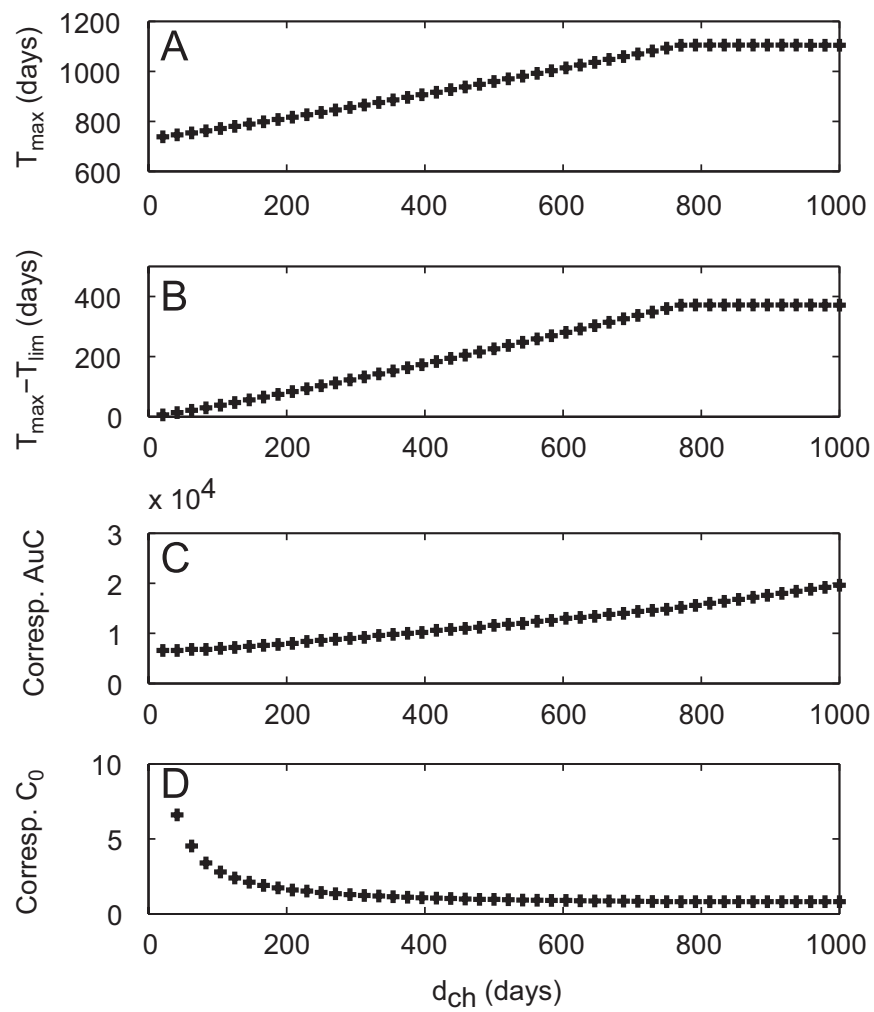

Fig. 6. Plot A shows the maximum survival time, $T_{\max }$, on varying the level of area under curve, $A u C$, for a range of chemotherapy durations $d_{c h}$. Plot $\mathrm{B}$ gives $T_{\max }-T_{\text {lim }}$, where $T_{\text {lim }}$ denotes the limit of the survival time as the area under curve, $A u C$, tends to infinity. This therefore shows the difference in the survival time due to the most judicious level of dosing compared to the theoretical maximum dose. Plots $C$ and $D$, respectively, show the area under curve and the chemotherapeutic cell kill, $C_{0}$, corresponding to the point where the survival time is maximised.

In the following, we vary the number of chemotherapy doses, denoted $N_{c y c}$, the drug cell kill, $C_{0}$, and the rest period between the doses, denoted $t_{\text {rest }}$. For definiteness, the duration of each administration is taken to be three days, but the choice of this value does not affect the qualitative form of the model's results.

Fig. 7 shows how the survival time varies with the rest phase duration $t_{\text {rest }}$, and the number of doses $N_{c y c}$, for three different, fixed, values of the drug cell kill $C_{0}$. These results are extended in Fig. 8; firstly, there is a plot of the maximum survival time, $T_{\max }$, with respect to variations of the rest phase, for each fixed value of the number of drug doses, $N_{c y c}$, and the cell kill $C_{0}$. Let $T_{0}$ denote the survival time for the limit of no rest phase; $T_{\max }-T_{0}$ is plotted, which shows the difference between the most judicious choice of rest phase and the most extreme dose densing protocol. In addition, the rest phase at which $T_{\max }$ occurs is depicted, showing that the optimal rest phase is, in large regions of parameter space, of significant duration.

Note from Fig. 7 that when a tumour cannot be eliminated, and the level of chemotherapeutic cell kill is held fixed, then protocol escalation by reducing the rest phase duration and increasing the number of cycles can lead to equivocal results. Similarly, dose intensification, by increasing the chemotherapeutic cell kill keeping other protocol parameters fixed, also can lead to substantial reductions or increases in survival time.

However, from Fig. 8, when the chemotherapeutic cell kill, rest phase duration and number of cycles are all treated as degree of freedoms with which to maximise the survival time, there are large regions of parameter space where dose densification is not optimal.
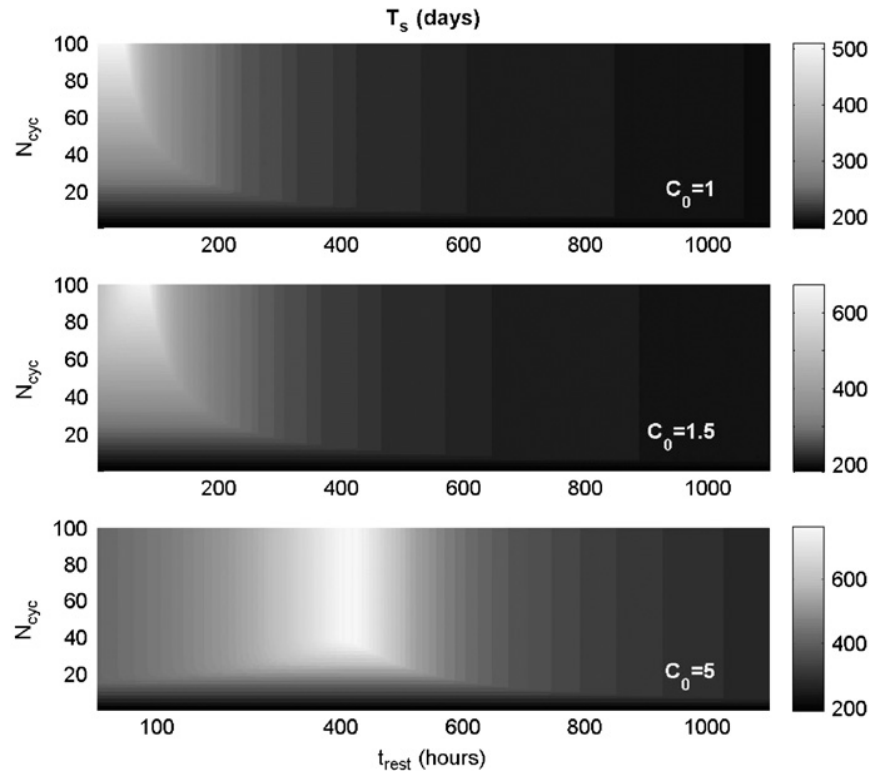

Fig. 7. Testing dose densification for palliative protocols. Predictions for the survival time, $T_{s}$, as a function of the rest phase duration, $t_{\text {rest }}$, and number of drug doses $N_{c y c}$ for three fixed rates of cell kill $C_{0}$.

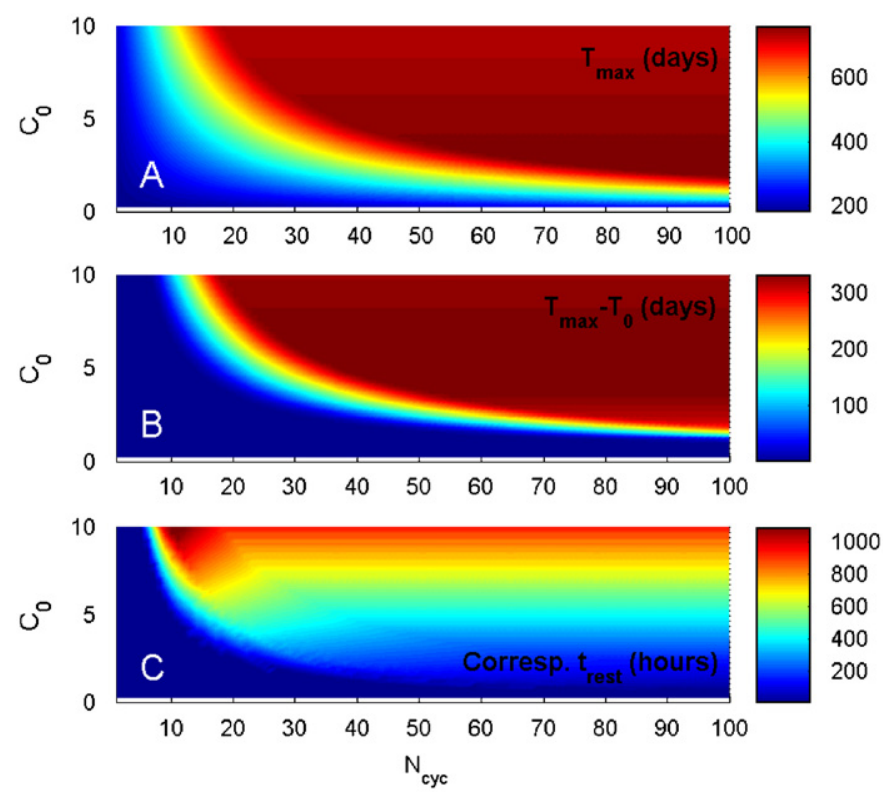

Fig. 8. Testing dose densification for palliative protocols. Plot A gives the maximum of the survival time with respect to variations of the rest phase, for each fixed value of the number of drug doses, $N_{c y c}$, and the cell kill $C_{0}$. Plot B similarly depicts the difference between this maximum of the survival time and the survival time in the limit that the rest phase tends to zero. Plot $C$ gives the rest phase corresponding to the maximum survival time.

The effect of varying the tumour cell number at chemotherapy initiation, $N_{c h}$, in the current context is explored in Fig. 9. Here the rate of cell kill $C_{0}$ is fixed; for each value of $N_{c h}$ and $N_{c y c}$, the rest phase between the doses is varied and the maximum survival time, $T_{\max }$, found as shown in Fig. 9A. The difference between $T_{\max }$ and the survival time in the absence of rest phases, denoted $T_{0}$, is plotted in Fig. 9B. The rest phase which yields the maximal survival time is depicted in Fig. 9C. The sharp cut off in survival time as the tumour burden increases is due to the fact the 

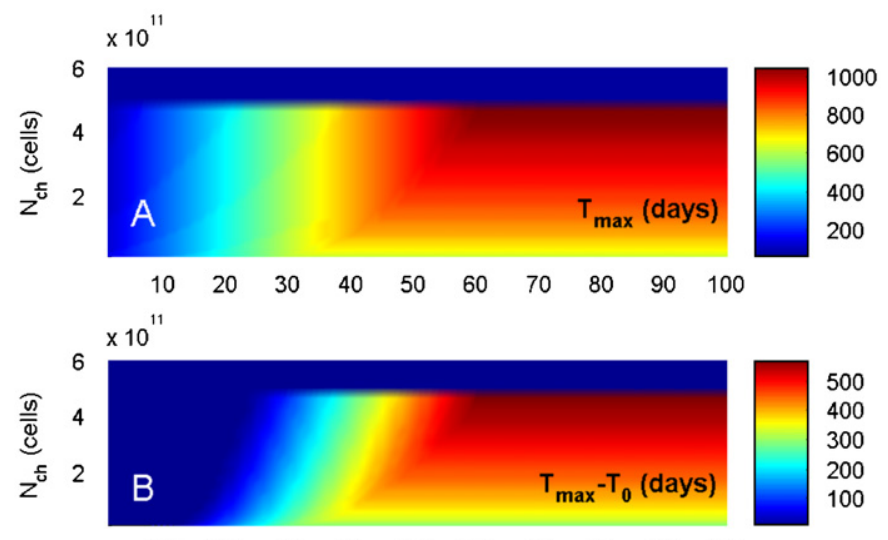

$\begin{array}{llllllllll}10 & 20 & 30 & 40 & 50 & 60 & 70 & 80 & 90 & 100\end{array}$

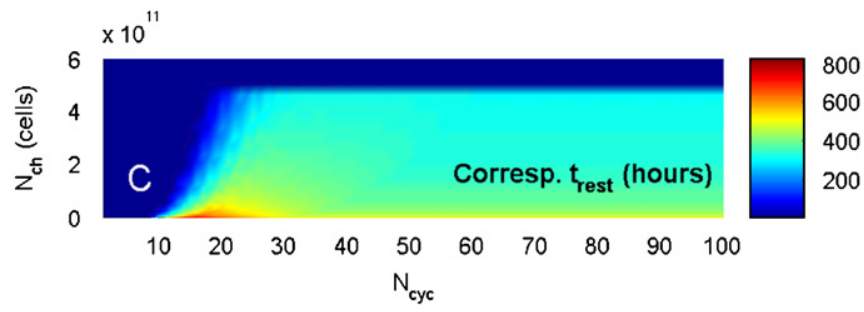

Fig. 9. The effects of variation of the initial tumour size in the dose densification context. For all these plots the cell kill is fixed at $C_{0}=5$. Plot $A$ gives the maximum of the survival time with respect to variations of the rest phase, for each fixed value of the number of drug doses, $N_{c y c}$, and tumour cell number at the chemotherapy start time, $N_{c h}$. Plot B gives the difference between the maximal survival time and the survival time in the limit of maximal dose densing, where the rest phase tends to zero. Plot $\mathrm{C}$ gives the corresponding value of the rest phase which maximises the survival time.

chemotherapy starts too late for the initial stages of treatment to prevent the tumour reaching its fatal size.

In summary, we can observe that protocol escalation need not yield significant improvements especially when given sufficient freedom to vary the scheduling parameters. In addition, the model predicts that survival time increases with delayed chemotherapy, at least providing the subsequent increased chemotherapy dosing and tumour size do not exceed tolerable limits.

\subsubsection{Dose densification for marginally incurable tumours}

It is of particular interest to highlight the effect of dose densification for a tumour that is only just incurable, as this is where it is most likely that a dose dense regime may be administered and fail to eradicate the tumour. A tumour will be considered "cured" when the cell number falls below a threshold level, denoted by $N_{\text {cure }}$ which we take to be 400 cells. Actual cure may have to be effected by other mechanisms given drug resistance. One possible example is a subsequent administration of a second drug or treatment; another is immunosurveillance (Liu et al., 2008).

Since here we are considering a potentially curative regimen, rather than palliation, a number of parameters are adjusted, as summarised in Table 2. In particular, we consider a scenario with a smaller tumour load at the chemotherapy initiation, and a less resilient tumour, that is a lower mutation rate to resistance. Thus, the therapy starting point is taken to be around $4 \times 10^{7}$ cells rather than the earlier reference point of $10^{10}$ cells; the mutation rate, and back mutation rate per cell cycle are given by $\tau_{1}=\tau_{2}=2 \mathrm{e}-7$; see the Appendix for further details.

We consider an initial tumour load of $N_{c h}=4 \times 10^{7}$ cells, plus a protocol with a rest phase, denoted $t_{\text {rest }}$, which intersperses a given number of administrations of a chemotherapeutic, denoted
Table 2

A list of representative, reference, parameters which are utilised, but differ, in the simulations of the marginally incurable tumour.

\begin{tabular}{|c|c|c|c|}
\hline Symbol & Represents & Value & Comment \\
\hline$\tau_{1}$ & $\begin{array}{l}\text { Mutation to resistance } \\
\text { per cell cycle }\end{array}$ & $2 \times 10^{-7}$ & $\begin{array}{l}\text { Goldie and } \\
\text { Coldman (1998) } \\
\text { and Appendix }\end{array}$ \\
\hline$\tau_{2}$ & $\begin{array}{l}\text { Back-mutation to } \\
\text { sensitivity per cell cycle }\end{array}$ & $2 \times 10^{-7}$ & $\begin{array}{l}\text { Goldie and } \\
\text { Coldman (1998) } \\
\text { and Appendix }\end{array}$ \\
\hline$N_{c h}$ & $\begin{array}{l}\text { Number of tumour cells } \\
\text { at treatment initiation }\end{array}$ & $\sim 4 \times 10^{7}$ cells & $\begin{array}{l}\text { Micrometastatic } \\
\text { tumour }\end{array}$ \\
\hline$N_{\text {cure }}$ & $\begin{array}{l}\text { Tumour cell burden } \\
\text { constituting cure }\end{array}$ & 400 cells & Cure threshold \\
\hline
\end{tabular}

The parameter, $N_{\text {cure }}$ is also introduced. Refer to the Appendix for further details of the parameter estimation.

$N_{c y c}$. Each administration is taken to be of three days' duration. We firstly determine the rate of cell kill, $C_{0}$, for which the dose dense limit of such a protocol, with no rest phases, only just induces cure. By this we mean the global minimum of the total tumour cell number for all time is equal to $N_{\text {cure. }}$. The value of $C_{0}$ that we find is, of course, dependent on the given value of $N_{c y c}$, and is plotted in Fig. 10A. The parameter values for these results, and those below, which are not listed in Table 2 are given in Table 1; additional details can be found in the Appendix.

To ensure the tumour is incurable, but only just, we subsequently simulate, for each value of $N_{c y c}$ and the value of $C_{0}$ plotted in Fig. 10A, a tumour with a slightly larger initial tumour cell number. Thus $N_{c h}$ now satisfies

$0<\frac{N_{c h}}{4 \times 10^{7}}-1 \ll 1 ;$

the simulation is otherwise unchanged. Consequently, not even the most extreme dose densing, corresponding to the limit of the rest phase tending to zero, can bring the tumour cell number below the cure threshold. If the chemotherapeutic cell kill level were to be raised above $C_{0}$ plotted in Fig. 10A, cure would be achieved, by construction, so this is excluded given the context of a failed curative attempt.

We do assume though that $C_{0}$ corresponds to a tolerable, and thus maximally tolerable, dose. Furthermore, given maximal cell kill is the intention of the dose densing regimen, and thus the level of cell kill is intended to be as high as possible, we do not reduce the chemotherapeutic cell kill level from the values plotted Fig. $10 \mathrm{~A}$ in the subsequent results.

In Fig. $10 \mathrm{~B}$ each point in the plot gives the maximal survival time as the rest phase is varied in a simulation of the marginally incurable tumour. The difference between the maximum survival time and the survival time in the dose densing limit of no rest phase is also plotted in Fig. 10C. Finally, the value of the rest phase which induces the maximal survival time is given in Fig. 10D.

The shading in this figure illustrates where a wide range of rest phases produce survival times very close to the maximum survival times. Thus, while we have plotted the rest phase which yields the maximum, one cannot infer significant information about the optimal rest phase other than survival times are relatively insensitive to the choice of the rest phase in this region of parameter space.

One can observe the model prediction that when there is a low number of drug administration cycles, dose densing does not substantially influence survival times for a marginally incurable tumour. However, should one consider larger numbers of cycles, the loss of survival time induced by dose densing on a marginal failure of chemotherapy is predicted to be significant. The precise number of administrations that is required before dose densing is 
A

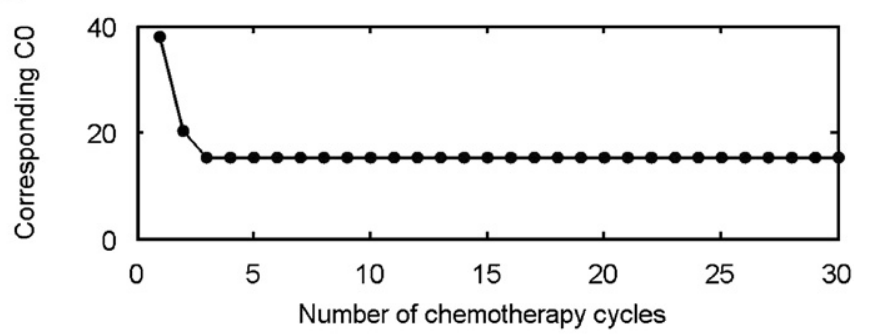

B

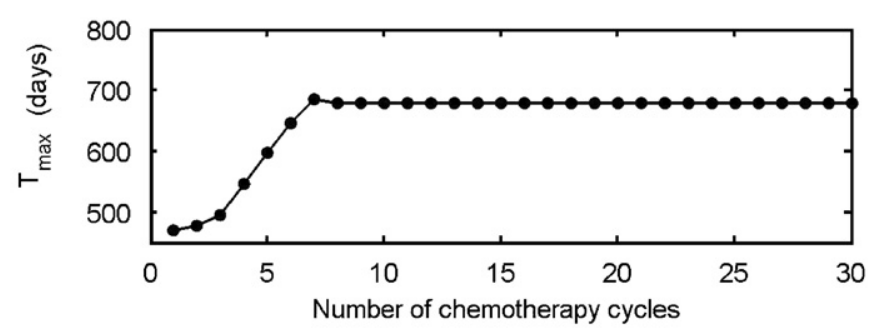

C

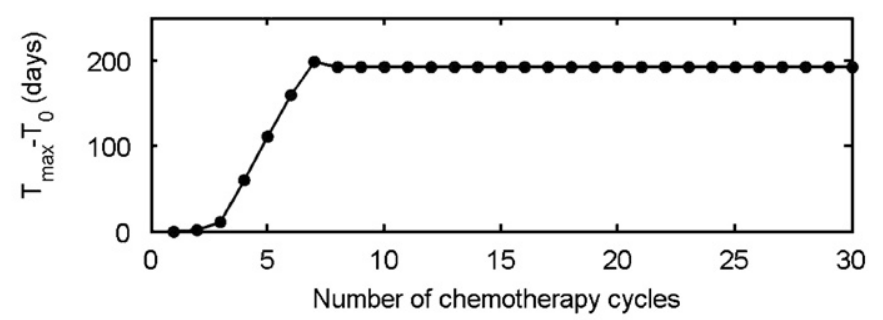

D

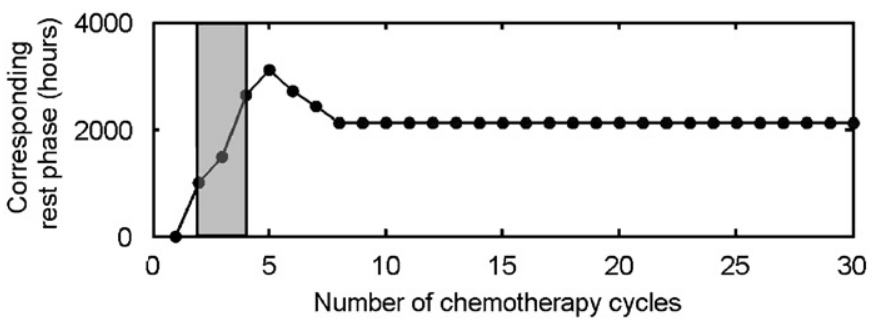

Fig. 10. Dose densification for a marginally incurable tumour; $\tau_{1}=\tau_{2}=2 \times 10^{-7}$, $N_{\text {cure }}=400$ cells, $N_{c h} \sim 4 \times 10^{7}$. The duration of an individual dose administration is three days; see text for further details on the parameter values. Plot A gives the minimum value of chemotherapy cell kill, $C_{0}$, which reduces the tumour cell burden to $N_{\text {cure }}$, as a function of the number of drug administrations, $N_{c y c}$. Graph B shows the maximum survival time, $T_{\max }$, with respect to the rest phase duration, for the marginally incurable tumour, with the tumour burden at the start of therapy as described in the text. Plot $C$ gives the difference between the maximal survival time and the survival time for no rest phase, $T_{0}$, while plot $\mathrm{D}$ gives the rest phase which maximises survival. See text for further details of the shading in the latter.

sub-optimal depends on the details of the protocol (results not shown); the above-mentioned qualitative observations are nonetheless robust both to the protocol details, such as administration duration, and variations in the parameters.

\section{Discussion}

\subsection{Continuous chemotherapy}

\subsubsection{Indefinite continuous protocols}

The results from indefinite continuous chemotherapy, as illustrated in Fig. 1, support the hypothesis that intermediate palliative doses of chemotherapy yield higher survival times compared to higher doses.

A more detailed examination of the cell population dynamics for drug doses around this optimum level reveals the reason for this behaviour; see Fig. 2. At lower doses, the chemotherapy cannot control the sensitive cell growth, resulting in the total cell number exceeding the fatal level relatively quickly. Increasing the cell kill, $C_{0}$, improves the control over the sensitive cells, thus increasing the survival time, until it reaches the maximum. At this optimal value of cell kill, the balance between controlling the sensitive cells and maintaining enough sensitive cells to restrict the growth of the resistant compartment via competition is such that the tumour burden remains below the critical level for the longest time.

Although the detrimental effect of maximal dose intensification on survival time in Fig. 3 is most pronounced for larger tumours, it is still predicted to result in significantly shorter survival for protocols initiated with smaller tumours.

In Fig. 4, it can be also seen that the survival time can be longer when the chemotherapy is started later and the initial tumour is larger, but only if the level of chemotherapy is higher. On first inspection, this suggests that chemotherapy should be delayed and a stronger dose administered. The mechanism is that the delay in therapy allows more sensitive cells to accumulate, so they are able to restrict the resistant cell population for longer, though a more aggressive chemotherapy dose is subsequently required. This behaviour does need to be viewed critically though, given the loss of model validity and the quality of life, due to the impact of tumour induced morbidity and chemotherapeutic toxicity at large delays of the chemotherapeutic start time.

Nonetheless, one does have the prediction that proactively starting treatment early for a cancer which is ultimately incurable due to drug resistance, as may happen in the adjuvant setting or with cancer screening, can adversely skew survival curves.

\subsubsection{Finite continuous protocols}

Where the treatment duration is continuous, but of a fixed and finite duration, Fig. 5 shows that the survival time rises to a maximum and then decreases as the area under curve is increased. This again supports the "less is more" concept that less intense chemotherapy in palliation can lead to improved results. This result is also observed to be significantly more pronounced for longer treatment duration; analogously, on considering the chemotherapy duration as an adjustable parameter, our results also reveal that the survival time can be higher for longer, less intense protocols.

\subsection{Protocols with rest phases}

In Fig. 7 we consider protocols with a given number of drug administrations, interspersed with rest phases, for three fixed values of chemotherapeutic cell kill. Where the survival time rises as the rest phase is lengthened, less dense schedules lead to an improved outcome, as in Fig. 7C. This occurs in an analogous way to the previous sections, where dose densing leads to sensitive cell death and thus less competitive control over resistant cells. However, for lower drug cell kill, as in Fig. 7A, Norton and Simon's prediction for dose densification holds, as the survival time only decreases as the rest phase increases. Here shorter rest phases are required in order to administer sufficient cell kill to control the sensitive population.

Figs. 8A and $\mathrm{B}$ show that when the chemotherapeutic cell kill and the number of cycles are fixed, the survival time is maximised by dose densing only for protocols which are either short or which have relatively low levels of chemotherapeutic cell kill. Most 
importantly, these figures reveal that on taking cell kill, rest phase and number of drug administrations as adjustable parameters the optimal results do not coincide with the most intense or dense protocol. Thus, again, "less is more" in the general palliative setting and protocol escalation does not provide a means of universally, or even typically, finding optimal outcomes in contrast to the concepts introduced by Norton and Simon (1976, 1986).

Similarly to the continuous chemotherapy case, Fig. 9 shows that delaying the chemotherapy start time in the dose densification context can lead to increased survival time, especially for longer protocol durations. Whereas the higher survival times in the previous case were achieved with higher rate of cell kill (see Fig. 4), here the rate of cell kill is fixed and the higher survival times are achieved with shorter rest phases between doses. Again this is due to a higher number of sensitive cells being present to restrict the growth of the resistant cells preventing the early emergence of near total tumour resistance. As previously, this aspect of the model's behaviour must be interpreted critically, especially at high levels of tumour burden. Nonetheless, it again illustrates a modelling prediction that very early palliation of an aggressive tumour can have a detrimental effect on survival time.

It is of particular interest to consider a marginally incurable tumour as this is a likely scenario for the implementation of a dose dense regimen that fails to induce tumour eradication. In Fig. 10B, whenever the difference between the maximum survival time and the survival time with no rest phases, is equal or close to zero, the maximal dose dense schedule with no rest phases is optimal or close to optimal. As with all predictions that protocol escalation is an appropriate strategy, it is qualified by assumption that dose densification does not lead to intolerable toxicity.

Inspection of Fig. 10 for larger numbers of drug administrations, also shows that dose densification can readily have a detrimental effect on the patient's survival time in the absence of tumour eradication, which would adversely skew modelling predictions of the protocols' survival curves. This does have to be balanced against the benefits in terms of an increased probability of cure that Norton and Simon's modelling predicts will emerge from protocol escalation.

\subsection{General discussion}

As previously remarked, the modelling concepts presented by Norton and Simon $(1976,1986)$ and Norton (1998, 1997, 2001), which generally advocate protocol escalation, contrast the modelling predictions of Hahnfeldt et al. (2003). The latter, based on exponential growth models, suggests that regimens based upon maximally tolerated chemotherapeutic doses are often not the most appropriate means of tumour treatment. Here, we have focussed on the setting where the chemotherapeutic cannot control tumour growth indefinitely with a model similar to Hahnfeldt et al. apart from the inclusion of Gompertzian growth. Thus the modelling incorporates the mechanisms underlying the studies by both groups and can therefore be used to consider which mechanisms, and thus which conclusions, are most appropriate in the current setting.

Gompertzian dynamics can have a substantial effect for protocols with rest phases. While protocol escalation is inappropriate for continuous infusions, there are regions of parameter space for a marginally incurable tumour where dose densification does not reduce survival times extensively should the attempted cure fail. Such regions are generally restricted to when the tumour is marginally incurable (and thus in practice there is a significant probability of cure) and the protocol is relatively short and intense. Given from the studies of Norton and Simon that dose densification can improve the probability of cure in such circumstances, it is clearly an appropriate strategy in this setting given tolerable toxicity.

However, in contrast, curative attempts involving sufficiently protracted protocols are predicted to have survival times in the absence of cure which are adversely affected by dose densification. This would severely and adversely skew modelling predictions of survival curves due to the sub-population who were not cured. Thus, one cannot draw definitive conclusions about whether protocol escalation is appropriate within the current modelling study. However, it is clearly illustrated that the modelling of dose densing strategies should not only consider the probability of cure, but also whether survival times are greatly reduced for failed protocols before drawing conclusions.

In the palliative setting, less is indeed more for chemotherapy and, for general protocols, protocol escalation leads to poorer outcomes in concordance with the modelling of Hahnfeldt et al. (2003). More generally, the model presented here reconciles the two competing concepts of Norton and Simon $(1976,1986)$ and Norton $(1998,1997,2001)$ and Hahnfeldt et al. (2003) within a single framework. Our conclusions thus additionally demonstrate that both frameworks can be valid according to context.

In summary, this model is based on well founded approximations: Gompertzian tumour growth, log cell kill by chemotherapy and Luria-Delbruck mutation to resistance, with results that are robust to parameter variation. We have modelling predictions that palliative continuous chemotherapy achieves optimal results for intermediate rather than high dosage levels with later rather than earlier intervention (subject to tolerability) due to the effects of competition between resistant and sensitive cells. It has been shown that protocol escalation via dose densification or dose intensification, will not yield benefits in the clearly palliative setting given sufficient freedom in protocol scheduling. In addition, very early palliation is predicted to lead to lower survival times. Finally, dose densification for a marginally incurable tumour will often, but need not, adversely affect survival time and thus skew predictions for the survival curve, especially for protocols with sufficiently large numbers of drug administrations.

The relevance of these observations are highlighted by the tentative consideration of single agent dose densification for the ovarian palliative setting (Vasey, 2005). This modelling clearly indicates that the gains predicted by Norton-Simon type modelling should not necessarily be expected in the context of cell cycle phase non-specific drugs used in the ovarian setting, such as cisplatin (Gorczyca et al., 1993; Vasey, 2005).

Scheduling for colorectal palliation is also a current area of investigation, including the potential role of regional continuous infusions (Fallik et al., 2003). While the above modelling of continuous infusions is insightful, a direct application of the current study in the context of colorectal palliation highlights the need to model combination protocols in future work. This requires numerous generalisations of the current study, including a heterogeneous spectrum of tumour resistance, cell cycle phase specificity, multiple drugs and the difference between regional and systemic treatments, especially in terms of metastases.

\section{Acknowledgements}

This work was supported in part by the Engineering and Physical Sciences Research Council, UK (EPSRC GR/S72023/01). We are also grateful to Prof. D.J. Kerr for useful and stimulating discussions. We are grateful to an anonymous referee for helpful comments. 


\section{Appendix A. Additional details and parameter values}

\section{A.1. Initial conditions}

In the simulations the tumour is grown from an initial single, drug sensitive, seed with

$N(t=0)=N_{S}(t=0) \equiv N_{0}=1$

and

$N_{R}(t=0)=0$

prior to the start of the chemotherapy. The initial stages are not consistent with a continuum model; however, once the cell numbers are larger this does provide a representative initial distribution of sensitive and resistant cells for subsequent study. Furthermore, the general robustness of the modelling framework to parameter variations entails that the conclusions we draw are insensitive to the consequences of neglected fluctuations in these initial stages of the tumour development. Thus a more sophisticated treatment of the initial dynamics would be an unnecessary complication.

\section{A.2. Gompertzian growth curve parameters}

For colorectal cancer, the doubling time $T$ is approximately $96 \mathrm{~h}$ (Rew and Wilson, 2000), so $\alpha_{N_{0}}$, the instantaneous specific growth rate of the tumour at time $t=0$ can be calculated:

$\alpha_{N_{0}}=\frac{\ln 2}{T}=7 \times 10^{-3} \mathrm{~h}^{-1}$.

$N_{\infty}$ is the theoretical number of cells at which the tumour growth curve would saturate; however, this is beyond the tumour size considered to be fatal so would not be reached in reality. The diameter of this beyond-fatal tumour size is taken to be about $16 \mathrm{~cm}$, i.e. $N_{\infty}=2 \times 10^{12}$ cells. Thus we have

$N_{\infty}=N_{0} \exp \left(\frac{\alpha_{N_{0}}}{\beta}\right) \Rightarrow \beta=2.47 \times 10^{-4} \mathrm{~h}^{-1}$.

For the above value of $\beta$ note that an advanced, near fatal, tumour with $N \sim 4 \times 10^{11}$ cells has a doubling time of about 72 days, which is the correct order of magnitude to match observations that, in clinic, tumours can have doubling rates of the order of 100 days (Rew and Wilson, 2000).

By non-dimensionalising with respect to time, one can see that altering $\beta$ is equivalent to changing the units of time. The overall dynamics are otherwise unchanged, assuming protocol rest phases and drug administration durations are also scaled. Thus our conclusions hold for a family of values of $\beta$. For example, if we were to use the 12.5 days doubling time of ovarian cancer (Rew and Wilson, 2000), we would find $\beta$ is reduced; with a suitable rescaling of time, conclusions are unchanged.

\section{A.3. Tumour cell burden levels}

We take, as reference, a nominal initiation point for chemotherapy to be a tumour cell burden of $N_{i n t}^{0}=10^{10}$ cells, corresponding to a tumour of roughly $1.5 \mathrm{~cm}$ in radius which is in the range of tumour sizes reported for patients initiating palliative care for non-resectable colorectal liver metastases (Becker et al., 1999). However, in practice, this is likely to be variable, so we also consider the effects of adjusting this parameter in the main text. In the context of failed cure in this paper, we take a much smaller initial tumour, with $4 \times 10^{7}$ cells at the start of treatment, which corresponds to a micrometastatic tumour of about $2 \mathrm{~mm}$ in radius. Note that micrometastases are a therapeutic target, as they are often anticipated to be present following post primary tumour resection ultimately leading to recurrence (Midgley and Kerr, 1999, 2000). In all simulations, the fatal radius of the tumour is taken to be $N_{\text {crit }}=5 \times 10^{11}$ cells, which corresponds to a tumour of diameter of approximately $10 \mathrm{~cm}$.

\section{A.4. Mutation to resistance}

As mentioned in the main text, the interpretation of our modelling representation of mutation contains a subtlety. In particular, the rate of mutation is proportional to the growth rate as considered in previous modelling (Goldie and Coldman, 1998). However, the underlying principle for mutation is that it is due to genetic errors occurring during replication and thus, in the absence of further empirical information, the mutation rate should be proportional to the proliferation rate.

One can, nonetheless, readily consider the errors involved with our modelling framework. Denote the proliferative rate by $P$ and the inherent (drug independent) rate of cell loss by $L$ so that the inherent (drug independent) growth rate is $P-L$. Further, let

$\varphi \stackrel{\text { def }}{=} \frac{L}{P}$,

denote the cell loss factor, which is commonly measured in tumour cell kinetic studies. For humans $\varphi$ is typically observed to be greater than 0.5 with a median value of 0.77 in a large study of human tumours (see Hall and Giaccia, 2006 and references therein) and values around 0.9 are also reported (e.g. Wickramanayake et al., 1985).

In terms of model parameters, the mutation rates per cell cycle between the sensitive and resistant compartments are

$\frac{(1-\varphi) \tau_{1}}{\ln 2}, \quad \frac{(1-\varphi) \tau_{2}}{\ln 2}$

Assuming the mutation rate per cell cycle is constant, our modelling framework has a systematic error in that we are then forced to assume $\varphi$ is constant. While in occasional studies this has been observed to be the case, e.g. Looney et al. (1971), generally $\varphi$ increases with tumour size. In particular, in observations of human tumour xenografts $\varphi$ is observed to be at least 0.46 in the exponential phase of growth and at most 0.91 as the plateau phase is reached, with respective mean values of 0.56 and 0.85 , as reported by Wickramanayake et al. (1985). Thus, taking an intermediate value of $\varphi$ our mutation rates have an error of at most a factor of 2.4 for the extremal values of $\varphi$ reported by Wickramanayake et al. (1985) and about 1.7 for the mean values. Even if we assume no cell loss in the early stages of growth, an intermediate value of $\varphi$ will still be limited to a factor-three error.

However, the model conclusions are robust to changes in the mutation rate. Thus, by neglecting the effect of the variation of the cell loss fraction during tumour growth we have a much simpler model, which need not separately track cell loss and yet we have conclusions which are insensitive to such details. Thus our modelling simplification is entirely appropriate for this initial, proof of principle, study.

We proceed to estimate the parameters $\tau_{1}, \tau_{2}$. The rate of mutation per cell cycle has been estimated in the experimental literature to be of the order of $\mathrm{O}\left(10^{-7}-10^{-4}\right)$ (Goldie and Coldman, 1998), while

\section{$\frac{(1-\varphi) \tau_{1}}{\ln 2}, \quad \frac{(1-\varphi) \tau_{2}}{\ln 2}$}

give the mutation rate per cell doubling time in the model. Thus, in the palliative setting $\tau_{1}$ and $\tau_{2}$ are taken to be $10^{-6}$. Significantly less aggressive tumours, with $\tau_{1}$ and $\tau_{2}$ given by $2 \times$ $10^{-7}$ are considered in the context of failed cure; such an extreme 
lack of aggression is required for cure to be possible in the model. At the initiation point for the palliative setting, the number of resistant cells present is about $10^{5}$ given the above choice of mutation rates. Similarly, in the context of failed cure, the smaller tumour initiation point and the smaller mutation rate entails that there are about 140 resistant cells present, which is still sufficient to justify a continuum model.

\section{A.5. Final remarks}

The nature of this field of modelling entails that the parameter values we use, as detailed above, are invariably estimates and will vary between different drugs, tumours and patients. However, the qualitative structure of the dynamics we have observed is robust to variations in our estimates at least providing palliation, or failed cure according to context, is feasible. For example, rapid progression to death with effectively no scheduling dependence occurs when the chemotherapeutic is so weak as to have essentially no control whatsoever over the tumour. However, the characteristics of the dynamics we have illustrated, and the conclusions we drawn, are retained for sensible parameter space samplings where the chemotherapeutic can reasonably be considered as having a realistic palliative, or curative, effect according to context.

\section{References}

Aabo, K., Vindelov, L.L., Christenson, I.J., Spang-Thomsen, M., 1994. Low-dose chemotherapy delays relapse of a dominated and resistant sub-population in a heterogeneous human SCLC xenograft in nude mice. Int. J. Cancer 59, 394-399.

Becker, D., Honsler, J.M., Strobel, D., Hahn, E.G., 1999. Percutaneous ethanol injection and radio-frequency ablation for the treatment of nonresectable colorectal liver metastases: techniques and results. Langenbeck's Arch. Surg. 384, 339-343.

Bello, L., Carrabba, G., Giussani, C., Lucini, V., Cerutti, F., Scaglione, F., Landre, J., Pluderi, M., Tomei, G., Villani, R., Carroll, R.S., Black, P.M., Bikfalvi, A., 2001. Lowdose chemotherapy combined with an antiangiogenic drug reduces human glioma growth in vivo. Cancer Res. 61, 7501-7506.

Bertolini, F., Paul, S., Mancuso, P., Monestiroli, S., Gobbi, A., Shaked, Y., Kerbel, R.S., 2003. Maximum tolerable dose and low-dose metronomic chemotherapy have opposite effects on the mobilization and viability of circulating endothelial progenitor cells. Cancer Res. 63, 4342-4346.

Browder, T., Butterfield, C.E., Kraling, B.M., Shi, B., Marshall, B., O’Reilly, M.S., Folkman, J., 2000. Antiangiogenic scheduling of chemotherapy improves efficacy against experimental drug-resistant cancer. Cancer Res. 60, 1878-1886.

Brünner, N., Bronzert, D., Vindelov, L.L., Rygaard, K., Spang-Thomsen, M., Lippman, M.E., 1989. Effect on growth and cell cycle kinetics of Estradiol and Tamoxifen on MCF-7 human breast cancer cells grown in vitro and in nude mice. Cancer Res. 49, 1515-1520.

Citron, M., Berry, D., Cirrincione, C., Carpenter, J., Hudis, C., Gradishar, W., et al., 2002. Superiority of dose-dense (DD) over conventional scheduling (CS) and equivalence of sequential (SC) vs combination adjuvant chemotherapy (CC) for node-positive breast cancer (CALGB INT C9741). Breast Cancer Res. Treat. 76 (Suppl. 1), A15.

Citron, M.L., Berry, D.A., Cirrincione, C., Hudis, C., Winer, E.P., Gradishar, W.J., et al., 2003. Randomized trial of dose-dense versus conventionally scheduled and sequential versus concurrent combination chemotherapy as postoperative adjuvant treatment of node-positive primary breast cancer: first report of Intergroup trial C9741/Cancer and Leukemia Group B trial 9741. J. Clin. Oncol. 21, 1431-1439.

De Placido, S., Perrone, F., Carlomagno, C., Morabito, A., Pagliarulo, C., Lauria, R., Marinelli, A., De Laurentiis, M., Varriale, E., Petrella, G., et al., 1995. CMF vs alternating $\mathrm{CMF} / \mathrm{EV}$ in the adjuvant treatment of operable breast cancer. A single centre randomised clinical trial (Naples GUN-3 study). Br. J. Cancer 71, 1283-1287.

Fallik, D., Ychou, M., Jacob, J., Colin, P., Seitz, J.F., Baulieux, J., Adenis, A., Douillard, J.Y., et al., 2003. Hepatic arterial infusion using pirarubicin combined with systemic chemotherapy: a phase II study in patients with nonresectable liver metastases from colorectal cancer. Ann. Oncol. 14, 856-863.

Gaffney, E.A., 2004. The application of mathematical modelling to aspects of adjuvant chemotherapy scheduling. J. Math. Biol. 48, 375-422.

Gaffney, E.A., 2005. The mathematical modelling of adjuvant chemotherapy scheduling: incorporating the effects of protocol rest phases and pharmacokinetics. Bull. Math. Biol. 67, 563-611.

Goldie, J.H., Coldman, A.J., 1998. Drug Resistance in Cancer. Cambridge University Press, Cambridge (ISBN 0521482739).
Goldie, J.H., Coldman, A.J., Gudaskas, G.A., 1982. Rationale for the use of alternating noncross resistant chemotherapy. Cancer Treat. Rep. 66, 439-449.

Gompertz, B., 1825. On the nature of the function expressive of the law of human mortality, and on a new mode of determining the value of life contingencies. Philos. Trans. R. Soc. London 115, 513-585.

Gorczyca, W., Gong, J., Ardelt, B., Traganos, F., Darzynkiewicz, Z., 1993. The cell cycle related differences in susceptibility of HL-60 cells to apoptosis induced by various antitumor agents. Cancer Res. 53, 3186-3192.

Hahnfeldt, P., Folkman, J., Hlatky, L., 2003. Minimizing long-term tumor burden: the logic for metronomic chemotherapeutic dosing and its antiangiogenic basis. J. Theor. Biol. 220, 545-554.

Hall, E.J., Giaccia, A.J., 2006. Radiobiology for the Radiologist. Lippincott Williams \& Wilkins (ISBN 0781741513).

Jaffrezou, J.P., Chen, G., Duran, G.E., Kuhl, J.S., Sikic, B.I., 1994. Mutation rates and mechanisms of resistance to etoposide determined from fluctuation analysis. J. Natl. Cancer Inst. 86, 1152-1158.

Kerbel, R.S., Kamen, B.A., 2004. The anti-angiogenic basis of metronomic chemotherapy. Nat. Rev. Cancer 4, 423-436.

Laird, A.K., 1964. Dynamics of tumor growth. Br. J. Cancer 13, 490-502.

Law, L.W., 1952. Origin of resistance of leukaemic cells to folic acid antagonists. Nature 169, 628-629.

Liu, Z., Eltoum, I.A., Guo, B., Beck, B.H., Cloud, G.A., Lopez, R.D., 2008. Protective immunosurveillance and therapeutic antitumor activity of $\gamma \delta$ T cells demonstrated in a mouse model of prostate cancer. J. Immunol. 180, 6044-6053.

Looney, W.B., Mayo, A.A., Janners, M.V., Mellon, J.C., Allen, P., Salak, D., Morris, H.P., 1971. Cell proliferation and tumor growth in hepatomas. Cancer Res. 31 $821-825$.

Luria, S.E., Delbruck, M., 1943. Mutation of bacteria from virus sensitivity to virus resistance. Genetics 28, 491-511.

Man, S., Bocci, G., Francia, G., Green, S.K., Jothy, S., Hanahan, D., Bohlen, P., Hicklin, D.J., Bergers, G., Kerbel, R.S., 2002. Antitumor and anti-angiogenic effects in mice of low-dose (metronomic) cyclophosphamide administered continuously through the drinking water. Cancer Res. 62, 2731-2735.

Midgley, R., Kerr, D., 1999. Seminar in colorectal cancer. Lancet 353, 391-399.

Midgley, R., Kerr, D., 2000. ABC of colorectal cancer: adjuvant chemotherapy. Br. Med. J. 321, 1208-1211.

Midgley, R., Kerr, D., 2005. Bevacizumab current status and future directions. Ann. Oncol. 16, 999-1004.

Norton, L., 1997. Evolving concepts in the systemic drug therapy of breast cancer. Semin. Oncol. 24 (4 Suppl 10), S10-3-S10-10.

Norton, L., 1998. A Gompertzian model of human breast cancer growth. Cancer Res. 48, 7067-7071.

Norton, L., 2001. Theoretical concepts and the emerging role of taxanes in adjuvant therapy. The Oncologist 6, 30-35.

Norton, L., Simon, R., 1976. Tumour size, sensitivity to therapy and the design of treatment protocols. Cancer Treat. Rev. 61, 1307-1317.

Norton, L., Simon, R., 1986. The Norton-Simon hypothesis revisited. Cancer Treat Res. 70, 163-169.

Piccart, M.J., Biganzoli, L., Di Leo, A., 2000. The impact of chemotherapy dose density and dose intensity on breast cancer outcome: what have we learned. Eur. J. Cancer 36, S4-S10.

Piccart-Gebhart, M.J., 2003. Mathematics and oncology: a match for life. J. Clin. Oncol. 21, 1425-1428.

Rew, D.A., Wilson, G.D., 2000. Cell production rates in human tissues and tumours and their significance. Part II: clinical data. Eur. J. Surg. Oncol. 26, 405-417.

Sieber, M., Tesch, H., Pfistner, B., et al., 2002. Rapidly alternating COPP/ABV/IMEP is not superior to conventional alternating COPP/ABVD in combination with extended-field radiotherapy in intermediate-stage Hodgkin's lymphoma: final results of the German Hodgkin's Lymphoma Study Group Trial HD5. J. Clin. Oncol. 20, 476-484.

Simon, R., Norton, L., 2006. The Norton-Simon hypothesis: designing more effective and less toxic chemotherapeutic regimens. Nat. Clin. Pract. Oncol. 3, 406-407.

Simpson-Herren, L., Lloyd, H.A., 1970. Kinetic parameters and growth curves for experimental tumor systems. Cancer Chemother. Rep. 54, 143-174.

Siodlak, M.Z., Stell, P.M., Wilson, J.A., Green, J.A., Allison, R.S., Adlera, D., SquadrelliSaraceno, M., 1990. Alternating cisplatinum and VAC ineffective in end stage squamous cell carcinoma of the head and neck. J. Laryngol. Otol. 104, 631-633.

Sullivan, P.W., Salmon, S.E., 1972. Kinetics of tumor growth and regression in IgG multiple myeloma. J. Clin. Invest. 51, 1697-1708.

Summer, W.P., Handshumacher, R.E., 1973. The rate of mutation of L5178Y asparagine-dependent mouse leukemia cells to asparagine independence and its biological consequences. Cancer Res. 33, 1775-1779.

Tiffany, T.A., Theodoulou, M., Feigin, K., Patil, S., Lee Tan, K., Edwards, C., Dugan, U. Norton, L., Hud, C., 2008. Phase I study of a novel capecitabine schedule based on the Norton-Simon mathematical model in patients with metastatic breast cancer. J. Clin. Oncol. 26, 1797-1802.

Vasey, P.A., 2005. "Dose dense" chemotherapy in ovarian cancer. Int. J. Gynecol Cancer 15 (Suppl. 3), 226-232.

Wickramanayake, P.D., Loeffler, M., Klein, H.D., Groth, W., Wichmann, H.E., 1985 Growth dependent cell proliferation kinetics of a human malignant melanoma grown in nude mice. J. Cancer Res. Clin. Oncol. 110, 11-16.

Winsor, C.P., 1932. The Gompertz curve as a growth curve. Proc. Natl. Am. Soc. 18, $1-8$. 